\title{
Neuregelung zu Publikationsverzeichnissen bei DFG-Anträgen: Stellungnahme des DFG-Fachkollegiums Psychologie
}

\author{
Elmar Brähler, Joachim C. Brunstein, \\ Michael Diehl, Edgar Erdfelder, \\ Clemens Kirschbaum, \\ Ulman Lindenberger, Erich Schröger, \\ Sabine Sonnentag, Rolf Ulrich, \\ Hannelore Weber
}

Die DFG hat nach Beratung im Präsidium und Senat Neuregelungen zu den Publikationsverzeichnissen bei DFGAnträgen beschlossen. Im Folgenden wollen wir kurz die Neuregelungen vorstellen, die von Seiten der DFG damit verbundenen Ziele kommentieren und das aus unserer Sicht positive und negative Potential dieser Regelungen diskutieren.

\section{Die Neuregelung}

Die neuen Regelungen beinhalten folgende Punkte (siehe auch www.dfg.de/foerderung/formulare_merkblaetter):

1) Im Antragsteil ,Stand der Forschung/Vorarbeiten/ Bericht" ist allein der Antragstext die Bewertungsgrundlage. In knapper und präziser Formulierung soll der Text für sich genügend Aussagekraft besitzen und ohne Hinzuziehen weiterer Literatur evaluierbar sein. Hinweise auf eigene und fremde, publizierte und unpublizierte Arbeiten können in einem entsprechenden Verzeichnis aufgeführt werden, es besteht aber keine Leseverpflichtung der Gutachter und Gutachterinnen. Unpubliziertes ist im Original beizufügen.

2) In den projektspezifischen Publikationen dürfen nur eigene Publikationen als Forschungsergebnisse erscheinen. Die maximale Anzahl pro Jahr der Förderung beträgt bei Einzelpersonen zwei, die maximale Anzahl für mehrere Personen pro Jahr der Förderung drei Publikationen. Dabei gelten für neu beantragte Projekte die zur Förderung beantragte Zahl der Förderjahre, für Fortsetzungsanträge die abgelaufenen Jahre. Es dürfen nur bereits erschienene oder angenommene Arbeiten in fachüblicher Gliederung sowie Buchveröffentlichungen angegeben werden. Bei angenommenen Arbeiten sind das Manuskript und die Bestätigung der Herausgeber beizufügen.

3) Der Lebenslauf darf maximal fünf Publikationen enthalten. Dabei sollen die aussagekräftigsten (besten) Arbeiten aufgeführt werden.

Die Fachkollegien wurden nicht in den Prozess der Erstellung dieser Neuregelungen eingebunden. Im Nachhinein wurde jedoch von fast allen Fachkollegien der DFG einhellige Zustimmung zu den Beschlüssen geäußert. Das Fachkollegium Psychologie gehörte zu den wenigen Fachkollegien, die erhebliche Bedenken gegen die Neuregelungen äußerten und in einem Schreiben an den Präsidenten der DFG eine entsprechende Korrektur forderten. Unsere Bedenken beziehen sich vor allem auf die Beschränkung von maximal fünf Publikationen im Lebenslauf.

\section{Welche Ziele verfolgt die DFG mit den Neuregelungen?}

Die DFG verfolgt mit den Neuregelungen fünf Zielsetzungen. Grundsätzlich versteht sie die Beschränkungen in den Angaben eigener Publikationen als eine Reaktion auf Fehlentwicklungen, die sich durch den Zwang zur großen Zahl von Publikationen und das zunehmende Gewicht von Zitationsindizes und Impact-Faktoren (vor allem in einzelnen Fächern) kennzeichnen lassen. Sie will den quantitativen Publikationsdruck von den Wissenschaftlerinnen und Wissenschaftlern nehmen und die Wissenschaft ins Zentrum rücken. Die Neuregelungen sollen dabei vor allem dem wissenschaftlichen Nachwuchs zugute kommen und (so DFG-Präsident Kleiner in einem Antwortschreiben an das Fachkollegium Psychologie) sich als familienfreundlicher erweisen. Als ein weiteres Ziel wird angegeben, dass ein Antrag aus sich heraus verständlich sein soll und somit für die Gutachterinnen und Gutachter leichter lesbar wird. Schließlich soll der Fokus auf publizierte und angenommene Arbeiten gelenkt werden, um reellere Angaben zu erzielen.

\section{Wie sind die Neuregelungen aus der Sicht der Psychologie zu bewerten?}

Aus unserer Sicht sind im Hinblick auf die Bewertung der Neuregelungen zwei Aspekte zu unterscheiden: (1) Die Begutachtung der Anträge durch die von der DFG beauftragten Gutachterinnen und Gutachter einschließlich der durch die Fachkollegien vorgenommenen Bewertung der Begutachtung im Sinne einer Qualitätssicherung und (2) die ,,ideologische“ Einbettung dieser Neuregelungen, die in der Öffentlichkeit mit Schlagworten wie ,Qualität statt Quantität“, „Pfähle gegen die Publikationsflut in den Wissenschaften“" und „Paradigmenwechsel“" proklamiert wurden.

Bezüglich Punkt (1) sind die Vorteile der Maßnahmen, die eine Bewertung des Antrags sui generis ermöglichen, offensichtlich. Wir stimmen nachdrücklich der Zielsetzung zu, dass ein Antrag für sich genommen überzeugen sollte, ohne dass Gutachterinnen und Gutachter die von 
den Antragstellern aufgeführten Publikationen im Detail rezipieren müssen. Aus unserer Sicht ist zur Zeit in zu vielen Anträgen die Qualität des geplanten Forschungsprogramms nicht leicht zu entnehmen. Hier könnten die Neuregelungen helfen. Ebenso begrüßen wir die explizite Klarstellung, dass nur tatsächlich veröffentlichte oder nachweisbar zur Veröffentlichung angenommene Arbeiten angegeben werden dürfen, womit „Luftnummern“ im Lebenslauf und in den projektspezifischen Publikationen künftig vermieden werden können. Im Berichtsteil eines Fortsetzungsantrags dürfen unpublizierte Arbeiten selbstverständlich angegeben und die zugehörigen Manuskripte beigefügt werden (siehe Punkt 1 der Neuregelung).

Weniger einsichtig ist aus unserer Sicht der Vorteil der Beschränkung des CV auf fünf Arbeiten. In den meisten Fällen ist die Information über die gesamte Publikationsliste zwar tatsächlich nicht relevant. Es gibt aber immer wieder Situationen, in denen diese Information zur Begründung von Entscheidungen unseres Erachtens herangezogen werden sollte. Dies geschieht aus unserer Erfahrung häufig zugunsten der Antragsteller und Antragstellerinnen. Ein Beispiel dafür ist der Fall, dass in einem Gutachten fehlende methodische Details im Antrag moniert werden (und der Antrag somit gefährdet wäre), aus der Veröffentlichungsliste jedoch klar hervorgeht, dass der Antragsteller oder die Antragstellerin die nötige Expertise besitzt. Auch zum Nachweis der Fähigkeit, eigene Arbeitsgruppen im Rahmen von Emmy-Noether-Programmen oder Heisenberg-Programmen leiten zu können, kann eine gute Publikationsliste nützlich sein. Im seltenen Fall, dass Anträge vergleichend-kompetitiv zu bewerten sind, kann die durch die Publikationsliste dokumentierte wissenschaftliche Produktivität den Ausschlag geben. Die temporäre Reduktion des wissenschaftlichen ,outputs' (z.B. Publikationen, Drittmittel, Tagungsaktivitäten), die auf Elternzeit oder andere Umstände zurückzuführen sind, sollte man unseres Erachtens durch geeignetere Maßnahmen berücksichtigen als durch die Beschränkung des CV auf fünf Publikationen.

Das Fachkollegium Psychologie sieht also keinen Nachteil darin, weiterhin den Gutachtern und Gutachterinnen sowie den Mitgliedern des Fachkollegiums die gesamte Publikationsliste zur Verfügung zu stellen, sondern erachtet dies eher als einen Vorteil. Hinzu kommt, dass aus Sicht des Kollegiums bei Förderentscheidungen bisher (meist) mit der erforderlichen Sensitivität vorgegangen worden ist, wobei auch die unterschiedlichen Publikationskulturen innerhalb einzelner Teildisziplinen der Psychologie berücksichtigt wurden.

Im Hinblick auf Punkt (2) stellen wir fest, dass mit den Neuregelungen und ihrer teils plakativen Begründung offensichtlich ein Thema angesprochen wurde, das auf unterschiedliche Empfindlichkeiten stößt. Dafür sprechen zumindest die Erfahrungen, die wir in den jüngsten $\mathrm{Ge}$ sprächen mit DFG-Repräsentanten aus verschiedenen Gremien und Fächern gesammelt haben. Insofern ist hier offenbar eine wichtige Debatte angestoßen worden. Der Slogan „Qualität statt Quantität" suggeriert jedoch einen negativen Zusammenhang, der sich empirisch nicht bestätigen lässt, wie beispielsweise Arbeiten von Keith
Simonton nahelegen, die eher auf einen positiven Zusammenhang hindeuten (wobei letztlich stets der Einzelfall zu bewerten ist). Wir bedauern, dass ein derart wichtiges Thema ohne fundierte Recherche aufgegriffen wurde, dabei zugleich eine Krankheit (zu hohe Bedeutung quantitativer Parameter) diagnostiziert und sofort ein Medikament (Beschränkung auf eine willkürlich festgesetzte Höchstgrenze) verordnet wurde. Auf Nachfrage durch das Fachkollegium Psychologie ist von Seiten des Präsidiums durchwegs mit anekdotischen Erfahrungen argumentiert worden, die die Selbstevidenz des beklagten Zustandes dokumentieren. Es ist uns mehrmals und bei verschiedener Gelegenheit versichert worden, dass eine Evaluierung dieser Maßnahmen nach zwei Jahren geplant sei, aber es konnten uns keine Kriterien genannt werden, die eine Entscheidung über Fortführen oder Beendigung der „Therapie" begründen werden.

Grundsätzlich sehen wir in den Neuregelungen keine Schwächung des hohen Stellenwerts qualitativ hochwertiger, international orientierter Publikationen. In Teilen erachten wir die Neuregelungen als begrüßenswert. Vor allem mit der Beschränkung von fünf Publikationen im CV sehen wir jedoch mögliche Nachteile verbunden, die unerwünschte Nebenwirkungen der verordneten Therapie darstellen. Diese Nebenwirkungen ergeben sich aus dem neu gesetzten Anreiz, mit maximal fünf Publikationen (den „super five“) zu glänzen. Dabei ist völlig offen, nach welchen Kriterien die ausgewählten fünf Publikationen in der Antragsbegutachtung bewertet werden. Wenn Auswahl und Bewertung erneut nach Impact-Faktoren und anderen quantitativen Parametern erfolgen, würde sich der gewünschte Paradigmenwechsel nicht nur nicht einstellen, sondern zöge zudem folgende Probleme nach sich:

- Die selbstgesteuerte Auswahl der fünf wichtigsten Publikationen wird den Wert dieser Arbeiten erhöhen und den Wert der nicht in den Kanon der ,super five“ aufgenommenen Arbeiten schwächen. Dies könnte zu einer Eigendynamik mit unerwünschten Folgen führen. Beispielsweise könnten die Projektleiter und Projektleiterinnen wieder mehr Interesse haben, selbst Erstautorschaften zu übernehmen (zu Lasten des wissenschaftlichen Nachwuchses). Darüber hinaus könnten die Anstrengungen nachlassen, Doktoranden und Doktorandinnen das Schreiben wissenschaftlicher Artikel zu vermitteln.

- Für die Nachwuchswissenschaftler und Nachwuchswissenschaftlerinnen entsteht nunmehr der Druck, sehr schnell möglichst hochrangige Publikationen vorzulegen, um bereits in ihren ersten DFG-Anträgen zu überzeugen. Die verbreitete Übungsphase anhand von kleineren, erfolgreich publizierten Arbeiten würde entfallen und sie könnten durch die wahrscheinlicher werdende Ablehnung ihrer Arbeiten in den hochrangigen Zeitschriften zu Beginn ihrer wissenschaftlichen Karriere entmutigt werden. Innerhalb von Nachwuchsgruppen könnte zudem ein verstärkter Kampf um die Erstautorschaft in absehbar hochrangigen Publikationen entbrennen. Die vermeintliche „Familienfreundlichkeit“ der Neuregelungen, die mit Blick auf die Vereinbarkeit von 
Wissenschaft und Familie den Druck zur großen Zahl nehmen soll, würde durch einen neuen Zwang zur möglichst zügigen Erstellung von „super five“ ersetzt. Angesichts des damit verbundenen Aufwandes geht die Familienfreundlichkeit zweifellos verloren.

- Nach unserer Ansicht ist die Falsifikation von Hypothesen ein wichtiges Vorgehen in der Wissenschaft. In den als qualitativ hochwertig eingestuften Zeitschriften kann man erfahrungsgemäß mit solchen Arbeiten meist wenig punkten, was zur Folge hat, dass die Darstellung akkumulierten Wissens auf der Grundlage publizierter Primärstudien zu verzerrten Ergebnissen führt. Dies ist zwar eine publikationsimmanente Fehlentwicklung, sie würde jedoch durch die Neuregelungen verstärkt werden.

- Mit der Fokussierung auf die „super five“ könnte eine implizite Abwertung der anderen Publikationen einhergehen, obschon diese durch bestehende Begutachtungsverfahren nicht minder qualitätsgeprüft sind. Zudem ist auch das Zeitschriftenranking nicht stabil, was zur Folge hätte, dass eine ehedem hochrangige Publikation aus der 1. Liga in die 2. Liga zurückfällt. Nach welchen Kriterien wählt hier ein Antragssteller oder eine Antragstellerin aus? Man sollte auch bedenken, dass qualitativ anspruchsvolle Arbeiten meist nicht aus dem Nichts entstehen, sondern in der Regel einen enormen Publikationsvorlauf erfordern. Man kann hier schwerlich Qualität gegen Quantität ausspielen.

Alle diese Aspekte sprechen aus unserer Sicht dafür, dass eine systematische Evaluation der Neuregelungen unabdingbar ist, um potentielle Fehlentwicklungen von Maßnahmen zu identifizieren, die eingeführt wurden, um Fehlentwicklungen zu korrigieren.

Trotz aller Kritik an diesen Details der Umsetzung (,super five“), ist der Tenor der Neuregelungen, den Fokus bei der Antragsbegutachtung noch stärker auf die Qualität der Anträge zu richten, begrüßenswert. Wir möchten an dieser Stelle die Diskussion über die Neuregelungen der Antragstellung dazu nutzen, alle Wissenschaftler und Wissenschaftlerinnen dazu aufzurufen, vermehrt Anträge zu stellen. Unser Appell richtet sich vor allem an den wissenschaftlichen Nachwuchs. Für ihn gilt ganz besonders, dass förderwürdige Anträge gute Ideen, überzeugende Vorarbeiten und ein plausibles Forschungsprogramm brauchen, aber kein ellenlanges Publikationsverzeichnis. 